\title{
Recent technologies, advances, and news in orthopedics
}

\author{
Carlos A. Acosta-Olivo* \\ Traumatology and Orthopedics Service, University Hospital "Dr. José Eleuterio González", Autonomous University of Nuevo Leon, Monterrey, Nuevo \\ Leon, Mexico
}

The field of orthopedic surgery has experienced an important advance over the past 15 years, having a stage of accelerated technological development, both in implants and surgical techniques, as well as in biotechnology to face the different challenges that pathologies demand.

Talking about low contact compression plates, the use of platelet-rich plasma (PRP), preserving the physis (growth zone in children), among others, is terms that are becoming more frequent in the various orthopedic forums worldwide.

At present, the results in the biotechnologies used for the treatment of different orthopedic conditions are variable. As an example, we have the use of PRP that, due to the different methods of obtaining it, as well as the variability in the number of platelets obtained in the same individual, has been the subject of multiple studies, some with better results than others. In this issue of Medicina Universitaria, we conducted a review of the different pathologies where their use has been documented. In the prevention of osteoarthrosis, both hip and knee, its major benefits have been seen. However, we had the opportunity to conduct a study in patients with delayed consolidation of humerus fractures, and we could see that patients who received PRP as coadjuvant showed earlier consolidation in a matter of weeks (19.9 weeks vs. 25.4 weeks) ${ }^{1}$.

Despite the multiple studies related to the use of PRP in orthopedics, new uses are still being found for this technology, as well as "buts" for its use, something that has been happening practically since its initial application. The techniques for its obtention have improved, and the indications have been refined to be able to establish the real utility of this biotechnology.

Another of the situations that have been taking more shape in orthopedic surgery is the care of pediatric patients with serious orthopedic injuries. This issue addresses the revision of a technique to preserve the area of the physis (area of growth in children) in patients with ligament injuries. Previously, the anterior cruciate ligament lesion in patients with open physis was to wait, until said that patient showed data of skeletal maturity (physis closure or physiological growth arrest) and thus be able to perform the repair of the cruciate ligament; with this waiting time, it was to try to avoid possible complications, such as the arrest of growth, which causes more problems for these patients, due to the deformities that could develop. At present, due to the increase of high-performance sport's patients and a better surgical technique, as well as the development of new implants, this type of ailments can be addressed once the diagnosis of the lesion has been made with excellent results for the patient.

In addition, a review of basic and current concepts regarding humeral diaphyseal fractures is presented since this type of fractures during this study has been able to obtain a consolidation, with adequate orthopedic management, close to $95 \%$. Much of the current literature, and sometimes, the same specialists and

\section{Correspondence:}

${ }^{*}$ Carlos A. Acosta-Olivo

E-mail: dr.carlosacosta @me.com
Available online: 30-09-2019

Date of reception: 02-06-2019

Date of acceptance: 13-06-2019 DOI: 10.24875/RMU.M19000034 www.medicinauniversitaria.org
Medicina Universitaria. 2019;21(3):90-91 CC BY-NC-ND license (http://creativecommons.org/licenses/by-nc-nd/4.0/). 
patients, are bombarded by the development of new implants, as well as the rapid incorporation into the daily activities of the affected person and opt for surgical treatment. It should be remembered that any surgical procedure is not exempt from possible complications, among which is an infection of the surgical site, bleeding, anesthetic complications, and others, and those that occur due to a fracture of the shaft of the humerus, such as the lesion of the radial nerve. This can be so devastating for the functionality of the patient's limb that it will need surgery for tendinous transpositions in case of permanent injuries. Due to this all, this review addresses the indications and options for orthopedic treatment, as well as the indications and surgical options.

The novelties in the surgical procedures, implants used and biotechnology developed in the area of orthopedics, must have precise indications to be able to obtain the success that we all want to achieve since the technological development without adequate knowledge of its application leads to failure and delay in progress. Hence, it is necessary to know, understand, and convince the real scope of current scientific advances to continue our continuous development.

\section{Ethical disclosures}

Protection of human and animal subjects. The authors declare that no experiments were performed on humans or animals for this study.

Confidentiality of data. The authors declare that no patient data appear in this article.

Right to privacy and informed consent. The authors declare that no patient data appear in this article.

\section{Reference}

1. Acosta-Olivo C, Garza-Borjon A, Simental-Mendia M, Vilchez-Cavazos F, Tamez-Mata Y, Peña-Martinez V, et al. Delayed union of humeral shaft fractures: comparison of autograft with and without platelet-rich plasma treatment: a randomized, single blinded clinical trial. Arch Orthop Trauma Surg. 2017;137:1247-52. 